\title{
Precise Information on Anisometropia
}

\author{
Partha Haradhan Chowdhury*1 and Brinda Haren Shah ${ }^{2}$ \\ ${ }^{1}$ M.OPTOM, ASSOCIATE PROFESSOR, PRINCIPAL, Department of Optometry, Shree Satchandi Jankalyan Samiti Netra Prasikshan Sansthan, Pauri, Affiliated to \\ Uttarakhand State Medical Faculty, Dehradun, India
}

${ }^{2}$ M.OPTOM, GUEST LECTURER, Department of Optometry, Shree Satchandi Jankalyan Samiti Netra Prasikshan Sansthan, Pauri, Affiliated to Uttarakhand State Medical Faculty, Dehradun, India

Received: 䟧: November 22, 2018; Published: ㄴㅔㅛ: November 27, 2018

*Corresponding author: Partha Haradhan Chowdhury, M.Optom, Associate Professor, Principal, Department of Optometry, Shree Satchandi Jankalyan Samiti Netra Prasikshan Sansthan, Pauri, Affiliated to Uttarakhand State Medical Faculty, Dehradun, India

Abstract

This paper describes about Anisometropia, its types with examples and its Treatment.

Keywords: Anisometropia, Types of Anisometropia

\section{Introduction}

Anisometropia is a binocular optical defect. It means it is a binocular phenomenon because refractive error of one eye is compared to other eye. In Anisometropia, refractive error of two eyes is different. If,

$$
\begin{array}{ll}
\text { i. } & \text { RE: }+0.25 \text { Ds }(6 / 6) \text {, } \\
\text { ii. } & \text { LE: +0.50Ds }(6 / 6)
\end{array}
$$

This is also considered as Anisometropia, but if difference is $+1.00 \mathrm{Ds}$, then it is considered as considerable Anisometropia. Eg.

$$
\begin{array}{ll}
\text { i. } & \text { RE: }+1.00 \text { Ds }(6 / 6) \\
\text { ii. } & \text { LE: +2.00Ds }(6 / 6)
\end{array}
$$

When the difference between refractive error of two eyes is $1.00 \mathrm{D}$, then Retinal size difference will be $2 \%$. Patient can tolerate upto $5 \%$ Retinal image difference between two eyes. It means two eyes Refractive error difference will be upto $2.50 \mathrm{D}$, but practically patient can tolerate upto 4.00D [1-3].

\section{Types of Anisometropia}
a) Simple Anisometropia
b) Compound Anisometropia
c) Mixed Anisometropia
d) Simple Astigmatic Anisometropia
e) Compound Astigmatic Anisometropia
f) Mixed Astigmatic Anisometropia

\section{Simple Anisometropia}

It is subdivided into Simple Myopic Anisometropia and Simple Hypermetropia Anisometropia. Here, one eye will be emmetropic and another eye is either Myopic or Hypermetropia.

Eg.

i. $\quad$ RE: Plano $(6 / 6)$

ii. LE: $-2.00 \mathrm{Ds}(6 / 6)$

This is a case of Simple Myopic Anisometropia.

i. RE: Plano (6/6)

ii. LE: +2.00Ds $(6 / 6)$

This is a case of Simple Hypermetropic Anisometropia.

\section{Compound Ansiometropia}

It is subdivided into Compound Hypermetropic Anisometropia and Compound Myopic Anisometropia. Here, different Refractive error is present between two eyes but both eyes have either plus power of minus power.

Eg:
a) Compound Hypermetropic Anisometropia
i. $\quad+2.00 \mathrm{Ds}(6 / 6)$
ii. +4.00Ds $(6 / 6)$
b) Compound Myopic Anisometropia 

i. $\quad$ RE: -2.00 Ds $(6 / 6)$
ii. LE: -4.00 Ds $(6 / 6)$

\section{Mixed Anisometropia}

Another name Mixed Anisometropia is " Antimetropia." Here, one eye is Hypermetropic and another eye is Myopic.
i. RE: +5.00Ds
ii. LE: -5.00Ds

\section{Simple Astigmatic Anisometropia}

It is subdivided into Simple Myopic Astigmatic Anisometropia and Simple Hypermetropic Astigmatic Anisometropia.

\section{a) Simple Myopic Astigmatic Anisometropia}

Here, one eye is Emmetropic and another eye is having Myopic Astigmatism
i. RE: Plano
ii. LE: $0.00 /-2.00$ Dcyl *90
b) Simple Hypermetropic Astigmatic Anisometropia

Here, one eye is Emmetropic and another eye is having Hypermetropic Astigmatism.

i. RE: Plano

ii. LE: $0.00 /+2.00$ Dcyl $^{*} 90$

ISSN: 2574-1241

DOI: $10.26717 / B J S T R .2018 .11 .002106$

Partha Haradhan Chowdhury. Biomed J Sci \& Tech Res

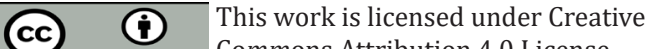

Commons Attribution 4.0 License

Submission Link: https://biomedres.us/submit-manuscript.php

\section{Compound Astigmatic Anisometropia}

Here, both eyes are astigmatic but of unequal degree, when it is Myopic then,

i. RE: -2.00 Dsph/ -2.00 Dcyl*90

ii. LE: $-4.00 \mathrm{Dsph} /-4.00 \mathrm{Dcyl}^{*} 90$

When it is Hypermetropic, then

i. $\quad$ RE: +2.00 Dsph / +2.00 Dcyl*90

ii. LE: + 4.00 Dshph/ +4.00 Dcyl *90

\section{Mixed Astigmatic Anisometropia}

i. $\quad$ RE: $+2.00 \mathrm{Dsph} /+4.00 \mathrm{Dcyl}^{* 90}$

ii. LE: -3.00 Dsph /-6.00 Dcyl *90

\section{Treatment}

Anisometropia is always treated with either Aspheric design of Spectacle lens or otherwise Contact Lenses. In case of high amount of Anisometropia, then Refractive surgery can be considered.

\section{References}

1. William J Benjamin (2006) Borish's Clinical Refraction ( $\left.2^{\text {nd }} E d n\right)$.

2. Theodore Grosvenor, Theodore P Grosvenor (2007) Primary Care Optometry ( $5^{\text {th }}$ Edn $)$.

3. Sir Stewart Duke Elder, David Abrams (1978) Duke-Elder's Practice of refraction.

$\begin{array}{ll}\text { BIOMEDICAL } & \text { Assets of Publishing with us } \\ \text { RESEARCHES } & \text { - Global archiving of articles } \\ & \text { - Immediate, unrestricted online access } \\ & \text { - Rigorous Peer Review Process } \\ \end{array}$

